\title{
Intravenous lipid emulsion for treatment of local anesthetic toxicity
}

\author{
This article was published in the following Dove Press journal: \\ Therapeutics and Clinical Risk Management \\ 2I September 2010 \\ Number of times this article has been viewed
}

\section{Caroline Kosh \\ April D Miller \\ Jill E Michels}

Department of Clinical Pharmacy and Outcomes Sciences, South Carolina College of Pharmacy, University of South Carolina Campus, Columbia, South Carolina, USA

Date of Preparation: I 4th July 2010

Conflict of interest: None declared
Correspondence: April D Miller South Carolina College of Pharmacy,

715 Sumter Street, Columbia,

SC 29208, USA

Tel + I 8037772265

Fax +I 803777 I943

Email millerad@sccp.sc.edu
Clinical question: Is intravenous lipid emulsion a safe and effective therapy for the reversal and treatment of local anesthetic toxicity?

Results: Systematic reviews, human case reports, and experimental animal studies have demonstrated the efficacy of intravenous lipid emulsion therapy in successfully reversing cardiac arrhythmias, cardiac arrest, and cardiac collapse seen with severe systemic local anesthetic toxicity. There are fewer data to support treatment of neurologic toxicities associated with local anesthetics.

Implementation: Intravenous lipid emulsion $20 \%$ should be available whenever patients receive large doses of local anesthetics in operating rooms and emergency departments. Various dosing protocols have been published in the medical literature. Although the dosing protocols are based on low-level evidence, a lack of major adverse events makes lipid emulsion an appropriate therapy for treating cardiotoxic symptoms induced by local anesthetics.

Keywords: intravenous lipid emulsion, local anesthetics

\section{Intravenous lipid emulsion in local anesthetic toxicity}

Definition: Local anesthetic overdose has been described as development of a variety of neurologic symptoms immediately following administration of local anesthetics. Symptoms are directly correlated with increasing local anesthetic blood concentrations. Depending on severity of overdose, progression to seizure and/or coma, cardiac arrhythmias, cardiac arrest, cardiovascular collapse, and even death, can occur.

Incidence: The rate of severe overdose with local anesthetic agents is very low. It is estimated that severe toxicity occurs in 7.5 to 20 per 10,000 peripheral nerve blocks and approximately four of every 10,000 epidurals administered. ${ }^{1}$ Specific patient risk factors, concurrent medications, location and technique of block, specific anesthetic, and total dose, along with time to detection and appropriateness of treatment, all influence the likelihood and degree of systemic toxicity from local anesthesia.

Economics: No published study has addressed the economic issues of treatment for local anesthetic toxicity using rescue lipid emulsion. Economic issues include increased operating room time, increased hospital and intensive care length of stay, cost of lipid treatment, and disability and missed work days for affected individuals.

Level of evidence used: Systematic review and clinical practice guidelines.

Search sources: PubMed, Cochrane Library, EMBASE, International Pharmaceutical Abstracts and relevant citations from retrieved articles.

Outcomes: From the patient perspective, the main outcomes are:

1. Effect on mortality

2. Residual disease or disability 
3. Effective reversal of cardiovascular and neurologic symptoms.

Consumer summary: Toxicity from local anesthetics (commonly called "caine" anesthetics) can be severe,

\section{The evidence}

\section{Does intravenous lipid emulsion infusion improve survival from local anesthetic toxicity?}

$\begin{array}{ll}\text { Systematic reviews: } & 1 \\ \text { Clinical practice guidelines: } & 2\end{array}$

There are no randomized, controlled clinical trials evaluating management of systemic local anesthetic toxicity due to rarity of the event and difficulty in obtaining informed consent for experimental medical interventions in acute critical illness.

The systematic review found 39 case reports in humans with return of spontaneous circulation following infusion of lipid emulsion cardiac arrest associated with local anesthetic. ${ }^{2}$ The review also cited a survival rate of $100 \%$ in a study of bupivacaine-induced cardiac arrest in dogs. Another study in rats showed a $30 \%$ increase in the lethal dose of bupivacaine administered after an intravenous lipid emulsion infusion. However, in a study of pigs receiving bupivacaine, there was a $100 \%$ return to spontaneous circulation in those treated with an epinephrine and vasopressin combination, but none in those treated with intravenous lipid emulsion.

\section{Is intravenous lipid emulsion effective in reversal of cardiovascular and neurologic symptoms associated with local anesthetic intoxication?}

The systematic review concluded that there is convincing anecdotal and experimental evidence that intravenous lipid emulsion is effective in treating toxicity caused by local anesthetics. ${ }^{2}$ Normalization of heart rate, blood pressure, and correction of QRS widening were documented with post including seizures and cardiac arrest. A fat solution (lipid emulsion) given in the veins can help reduce the side effects and prevent death and disability. intravenous lipid emulsion infusion. Electrocardiographic abnormalities were also corrected, including asystole conversion, correction of torsades de pointes, and achievement of normal sinus rhythm from ventricular tachycardia. Mental status improvements in both unpublished and published human case reports have been reported.

Clinical practice guidelines state that Intralipid ${ }^{\circledR} 20 \%$ has been shown to reverse cardiac arrest induced by local anesthetic in animal models and in human case reports, and its use has been reported in the treatment of life-threatening toxicity. It is recommended that intravenous lipid emulsion therapy be administered in all situations in which circulatory arrest attributed to local anesthetic toxicity is present, and considered in patients with cardiac symptoms other than cardiac arrest. ${ }^{3}$

Additional clinical practice recommendations cite lipid emulsion as a modality to mitigate cardiac toxicity seen with local anesthetic toxicity. ${ }^{4}$ Pancreatitis is a rare side effect associated with lipid emulsion therapy. However intravenous lipid emulsioninduced pancreatitis is related to cumulative dosing and is seen with long-term repeated use in other disease states.

\section{Conclusions}

There is sufficient evidence to support the efficacy of intravenous lipid emulsion in the treatment of toxic overdose from local anesthetics and reversal of associated cardiovascular symptoms. Human data are limited for its use in managing neurotoxicity. Controversy concerning optimal dosing, therapy duration, and delay of administration still exists, and further study is warranted. Additional data focusing on adverse events and long-term effects are also necessary.

\section{The practice}

\section{Potential pitfalls}

Avoid vasopressin, calcium channel blockers, beta-blockers, or local anesthetics. In patients with cardiovascular instability, avoid propofol because of the potential for hypotension.

\section{Management}

- Stop local anesthetic administration

- Basic or advanced life support is essential
- Focus on airway maintenance (with endotracheal intubation if needed)

- Give $100 \%$ oxygen, and ensure adequate lung ventilation

- Intravenous access should be established

- Seizure control should be obtained with benzodiazepines

\section{Assessment}

- Local anesthetic induced toxicity can occur up to 30 minutes following end of infusion 
- Patients should be monitored closely throughout, including neurologic and cardiovascular assessment

- Neurologic signs may be subtle or absent, and include: Excitation (agitation, confusion, muscle twitching, seizure) Depression (drowsiness, obtundation, coma, apnea)

Nonspecific (metallic taste, circumoral numbness, diplopia, tinnitus, dizziness).

Severe intoxication is most often indicated by cardiovascular signs, including hyperdynamic signs and symptoms (hypertension, tachycardia, ventricular arrhythmias) with progression to:

- Hypotension

- Conduction block, bradycardia, or asystole

- Ventricular arrhythmias

\section{Treatment}

Circulatory arrest

- Cardiopulmonary resuscitation and advanced cardiac life support, as indicated

- Manage arrhythmias (do not use lidocaine)

- Consider cardiopulmonary bypass in severe cases

Lipid emulsion efficacy may be reduced by high dose vasopressors $^{5}$

Initial intravenous bolus injection of $20 \%$ lipid emulsion $1.5 \mathrm{~mL} / \mathrm{kg}$ over one minute and start an intravenous infusion of $20 \%$ lipid emulsion at $0.25 \mathrm{~mL} / \mathrm{kg} /$ minute

- Lipid emulsion therapy administered via peripheral or central line

After five minutes, give two repeat boluses (maximum of three boluses) if cardiac stability has not been restored or adequate circulation deteriorates

- Leave five minutes between boluses

- Double infusion rate to $0.5 \mathrm{~mL} / \mathrm{kg} /$ minute if no clinical improvement or deterioration occurs at any time

- Continue infusion until clinically stable and adequate circulation restored or maximum dose of lipid emulsion $12 \mathrm{~mL} / \mathrm{kg}$ has been administered
- Without circulatory arrest, use conventional therapy to treat hypotension, bradycardia, or tachyarrhythmia, and consider intravenous lipid emulsion therapy

- Monitor patients overnight for any signs of cardiac toxicity.

\section{Indications for specialist referral}

- The acute nature of cardiac toxicity from local anesthetics complicates specialist referral and early treatment

- Once initial symptoms subside, patients should be monitored by a cardiologist or intensivist

- Professionals with expertise in cardiopulmonary bypass should be consulted for severe cases.

\section{Further reading}

Jamaty C, Bailey B, Larocque A, Notebaert E, Sanogo K, Chauny JM. Lipid emulsions in the treatment of acute poisoning: A systematic review of human and animal studies. Clin Toxicol (Phila). 2010;48:1-27.

http://www.aagbi.org/publications/guidelines/docs/la_toxicity_2010.pdf (Practice). Accessed July 15, 2010.

www.lipidrescue.org (Evidence/Practice). Accessed July 15, 2010.

\section{References}

1. Corcoran W, Butterworth J, Weller RS, et al. Local anestheticinduced cardiac toxicity: A survey of contemporary practice strategies among academic anesthesiology departments. Anesth Analg. 2006;103:1322-1326.

2. Jamaty C, Bailey B, Larocque A, Notebaert E, Sanogo K, Chauny JM. Lipid emulsions in the treatment of acute poisoning: A systematic review of human and animal studies. Clin Toxicol (Phila). 2010; 48:1-27.

3. Association of Anaesthetists of Great Britain and Ireland. Guidelines for the management of severe local anaesthetic toxicity. 2007. Available at: http://www.aagbi.org/publications/guidelines/docs/latoxicity07.pdf. Accessed July 15, 2010.

4. Neal JM, Bernards CM, Butterworth JFT, et al. ASRA practice advisory on local anesthetic systemic toxicity. Reg Anesth Pain Med. 2010;35:152-161.

5. Hiller DB, Gregorio GD, Ripper R, et al. Epinephrine impairs lipid resuscitation from bupivacaine overdose: A threshold effect. Anesthesiology. 2009;111:498-505.
Therapeutics and Clinical Risk Management

\section{Publish your work in this journal}

Therapeutics and Clinical Risk Management is an international, peerreviewed journal of clinical therapeutics and risk management, focusing on concise rapid reporting of clinical studies in all therapeutic areas, outcomes, safety, and programs for the effective, safe, and sustained use of medicines. This journal is indexed on PubMed Central, CAS,

\section{Dovepress}

EMBase, Scopus and the Elsevier Bibliographic databases. The manuscript management system is completely online and includes a very quick and fair peer-review system, which is all easy to use. Visit http://www.dovepress.com/testimonials.php to read real quotes from published authors. 